\title{
The effect of middle ear effusion on the inner ear condition in children
}

\author{
Katarzyna Pazdro-Zastawny ${ }^{A-F}$, Tomasz Zatoński ${ }^{\mathrm{E}, \mathrm{F}}$ \\ Department and Clinic of Otolaryngology, Head and Neck Surgery, Wroclaw Medical University, Poland \\ A - research concept and design; $B$ - collection and/or assembly of data; $C$ - data analysis and interpretation; \\ $D$ - writing the article; $E$ - critical revision of the article; $F$ - final approval of the article
}

Address for correspondence

Katarzyna Pazdro-Zastawny

E-mail: kasiapz@poczta.fm

\section{Funding sources \\ None declared}

\section{Conflict of interest}

None declared

\section{Acknowledgements}

We express our utmost gratitude to Professor Lucyna Pośpiech who provided advice and insight during the course of this research.

Received on March 26, 2019

Reviewed on April 5, 2019

Accepted on September 25, 2019

Published online on March 24, 2020

\begin{abstract}
Background. Otitis media with effusion (OME) is the most common cause of hearing impairment among children in developed nations. Middle ear (ME) fluid accumulation leads to progressive hearing impairment, usually of the conductive type. In some cases, mixed hearing loss associated with OME has been noted. It was reported that effusion in the ME has a negative impact on the vestibular system of the inner ear.
\end{abstract}

Objectives. The aim of this random-sample cohort study was to evaluate postural stability and the influence of ME drainage on vestibulospinal reflexes in children with OME, and to determine whether disturbances in the vestibular organ correlate with a sensorineural component in OME-related hearing loss.

Material and methods. The study group consisted of 53 children with bilateral OME who were treated with bilateral MEdrainage. The study group was divided into subgroups according to hearing loss. The control group consisted of 29 healthy children. Vestibular function and hearing evaluation were performed before and 4 weeks after drainage.

Results. A comparison of the stabilograms of the study group and the control group revealed elevated parameters in most of the tests. In the subgroup with mixed hearing loss, either before or after ME drainage, elevated stabilogram parameters were found in all tests. Posturography revealed vestibular system disturbances before and after ME drainage in the subgroup with mixed hearing loss, especially before ME drainage. The stabilogram parameters in the subgroup with conductive hearing loss after ME drainage were better in most tests in comparison to those before the procedure.

Conclusions. The presence of effusion in the ME has a negative effect on the inner ear. We highlight the importance of monitoring the condition of the vestibular system in all children with OME, especially in cases with mixed hearing loss and more advanced clinical stages of the disease.

Key words: vertigo, balance disorders, otitis media with effusion

Cite as

Pazdro-Zastawny K, Zatoński T. The effect of middle ear effusion on the inner ear condition in children. Adv Clin Exp Med. 2020;29(3):325-330. doi: 10.17219/acem/112601

DOI

10.17219/acem/112601

\section{Copyright}

Copyright by Author(s)

This is an article distributed under the terms of the

Creative Commons Attribution 3.0 Unported (CC BY 3.0)

(https://creativecommons.org/licenses/by/3.0/) 


\section{Background}

Otitis media with effusion (OME) is the most common cause of hearing impairment among children in developed nations. ${ }^{1-3}$ Middle ear (ME) fluid accumulation ${ }^{4-8}$ leads to progressive hearing impairment, usually of the conductive type. In some cases, mixed hearing loss associated with OME has been noted. ${ }^{9,10}$ It was reported that effusion in the ME has a negative impact on the vestibular system of the inner ear.,11-14

\section{Objectives}

The aim of this random-sample cohort study was to evaluate postural stability and the influence of ME drainage on vestibulospinal reflexes in children with OME and to determine whether disturbances present in the vestibular organ correlates with a sensorineural component in OME-related hearing loss.

\section{Material and methods}

\section{Material}

The study group consisted of 53 children (20 females and 33 males) aged $4-14$ years (mean age: 8 years; SD: 2.5 years) diagnosed with bilateral OME. Those children were treated with bilateral ME drainage.

The study group included children with recurrent or persistent bilateral OME persisting 3 months or longer after conservative treatment had proven ineffective, with a hearing level in the better ear of $25-30 \mathrm{dBHL}$ or worse, averaged at $0.5 \mathrm{kHz}$, $1 \mathrm{kHz}, 2 \mathrm{kHz}$, and $4 \mathrm{kHz}$ (or an equivalent dBA where $\mathrm{dBHL}$ was not available), and with type B tympanometry.

Children with a birth weight of less than 2,500 g; a history of neonatal asphyxia; congenital malformations of the external, middle or inner ear; temporal bone fracture; neurological diseases or any other serious illness (epilepsy); a history of meningitis; and a history of vestibulotoxic or ototoxic drugs were excluded from the study.

The study group was divided into the following subgroups according to hearing loss:

- conductive hearing loss (25 children: 14 boys and 11 girls) aged 4-11 years (average age: 7.76 years; SD: 1.8 years) and

- mixed hearing loss (28 children: 19 boys and 9 girls) aged $4-14$ years (average age: 8.18 years; SD: 2.85 years).

The subgroup with mixed hearing loss included children with more severe pathological changes, including retraction pockets or local atrophy of the tympanic membrane, or those with a past history of ME drainage and a disease lasting longer than 2 years.

The control group consisted of 29 healthy children (13 girls and 16 boys) aged 4-17 years (average age: 10 years;
SD: 3.8 years), who had been scheduled for a tonsillectomy operation in the Ear, Nose and Throat (ENT) Department. The children's medical history was obtained from their parents.

\section{Methods}

All of the children underwent a complete otoneurological examination. A detailed case history was collected, with particular attention paid to the current disease, previous ENT diseases, and the presence of vertigo and/or disequilibrium. The children and parents were asked whether the children had suffered from frequent falls; had difficulty riding a bicycle, or climbing or descending stairs; whether they disliked swings; had a tendency to bump into objects or to misjudge distances; experienced vertigo, dizziness, tinnitus, or disequilibrium; or whether they exhibited unexplained clumsiness, delayed gross motor development or recurrent headache.

Each child underwent a full physical otolaryngological examination. An assessment of ME status and the function of the ventilation tubes was performed using pneumatic otoscopy, performed by a certified otoscopist.

Tympanometry was performed using a Madsen Zodiac 801 tympanometer and done by a qualified audiologist prior vestibular testing. An audiometric evaluation using a Madsen OB 822 (Otometrics, Denmark) audiometer was performed by means of conventional audiometric methods in a soundproof room. The collected data included air conduction and bone conduction thresholds in each ear at frequencies of $0.5 \mathrm{kHz}, 1 \mathrm{kHz}, 2 \mathrm{kHz}$, and $4 \mathrm{kHz}$. The pure-tone average for bone conduction in each ear was calculated for each patient as the average of bone conduction thresholds at $0.5 \mathrm{kHz}, 1 \mathrm{kHz}, 2 \mathrm{kHz}$, and $4 \mathrm{kHz}$. The air-bone gap in the affected ear was calculated as the difference between air conduction and bone conduction thresholds at $0.5 \mathrm{kHz}, 1 \mathrm{kHz}, 2 \mathrm{kHz}$, and $4 \mathrm{kHz}$. Pure-tone audiometry and tympanometry was performed before vestibular testing. Audiometry was carried out before drainage and 4 weeks postoperatively. Tympanometry was only performed prior to ME drainage.

Vestibulospinal reflexes were evaluated through statoposturography using a Posturographer PE 62 Model 04 (Neurocom, Luxembourg). The diagnostic system consisted of an an IBM microcomputer (IBM Corp., Armonk, USA) with additional converters, a static posturographic $40 \times 40 \mathrm{~cm}$ platform with pressure sensors recording deflections in the range of $\pm 10 \mathrm{~cm}(0.05 \mathrm{~mm}$ accuracy), and a visual stimulator connected to a TV screen. In each case, a set of 3 tests was performed in order to evaluate static balance. Each child was tested under different conditions: test 1 was performed standing with eyes open (E-O); test 2 was performed standing on the platform with eyes closed (E-C); and test 3 was performed with eyes open and feet rested on the platform, the child observing a moving point of light, which reflects the current 
position of the center of gravity, and making slight movements of the body in order to self-correct their current body position (feedback test (F-T)). Three trials, lasting $30 \mathrm{~s}$ each, were performed for each condition. The field of developed area (FDA; the area described by the center of mass $\left[\mathrm{mm}^{2}\right]$ ) and the average sway velocity (ASV) $[\mathrm{mm} / \mathrm{s}]$ were analyzed.

Vestibular testing was performed by a technician who had no knowledge of each child's ear status. Vision tests were not carried out.

Children with bilateral OME were examined while hospitalized, before the bilateral insertion of ventilation tubes and 4 weeks after the procedure during their postoperative check-up.

Informed consent was obtained from all parents/legal guardians of individual participants included in the study. The study was conducted in accordance with the Declaration of Helsinki after obtaining approval from the local Bioethics Committee (approval No. KB-29/2005). One or both parents accompanied each child during all testing procedures.

Statistical analysis was carried out using Student's t-test to compare the average values of parameters before and after drainage. A p-value $<0.05$ was considered to be statistically significant for all tests. The statistical analysis was performed using STATISTICA v. 13.0 (StatSoft, Inc., Tulsa, USA).

\section{Results}

Otoscopic testing showed OME symptoms in all of the selected patients. Before the ventilation tubes were inserted, none of the patients complained of vertigo or balance disorders. In the study group, impedance audiometry showed bilateral type B tympanometry before $\mathrm{ME}$ drainage in all 53 children (100\%). Before drainage, puretone audiometry showed bilateral conductive hearing loss in the range of $40-50 \mathrm{~dB}$ in 25 children (47\%) and bilateral mixed hearing loss with an average air conduction threshold of 35-45 dB at high frequencies in 28 children (53\%).

Control pure-tone audiometry performed after ME drainage revealed:

- in the subgroup with conductive hearing loss - normal hearing in 22 children (88\%) and bilateral conductive hearing loss with cochlear reserve approx. $15 \mathrm{~dB}$ in 3 children (12\%), and

- in the subgroup with mixed hearing loss - a decrease or lack of cochlear reserve and bilateral high-frequency hearing loss in the range of 35-45 dB.

\section{Posturography}

A comparison of the stabilograms of the study group and the control group revealed elevated parameters for FDA and ASV in most of the tests. The findings showed statistically significant values $(\mathrm{p}<0.001)$ before ME drainage for FDA in all tests and for ASV with E-O and E-C in the study group. After the ME drainage procedure, there were statistically significant values for FDA with E-O and E-C (Fig. 1-4).

The stabilogram parameters were compared between subgroups, divided according to the group with hearing loss and the control group.

In the subgroup with mixed hearing loss, either before or after ME drainage, elevated stabilogram parameters were found for FDA and ASV in all tests in comparison with those of the subgroup with conductive hearing loss. Before drainage, there were statistically significant values $(\mathrm{p}<0.05)$ for FDA with E-O and E-C and for ASV with E-O. After ME drainage, statistically significant values $(\mathrm{p}<0.05)$ were found for FDA with the F-T (Fig. 5,6).

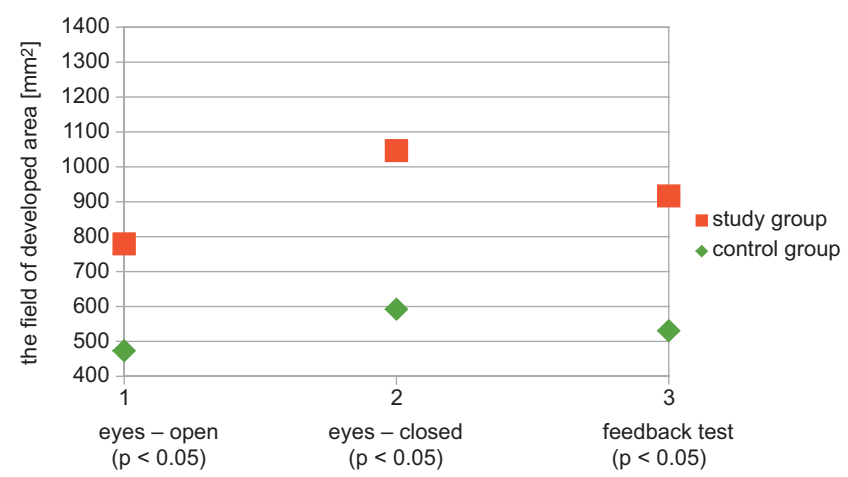

Fig. 1. The FDA in the study and control groups before ME drainage

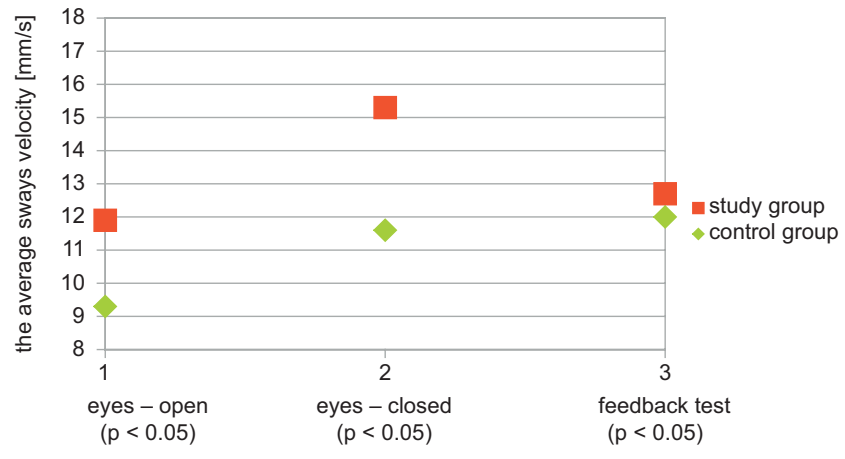

Fig. 2. The ASV in the study and control groups before ME drainage



Fig. 3. The FDA in the study and control groups after ME drainage 


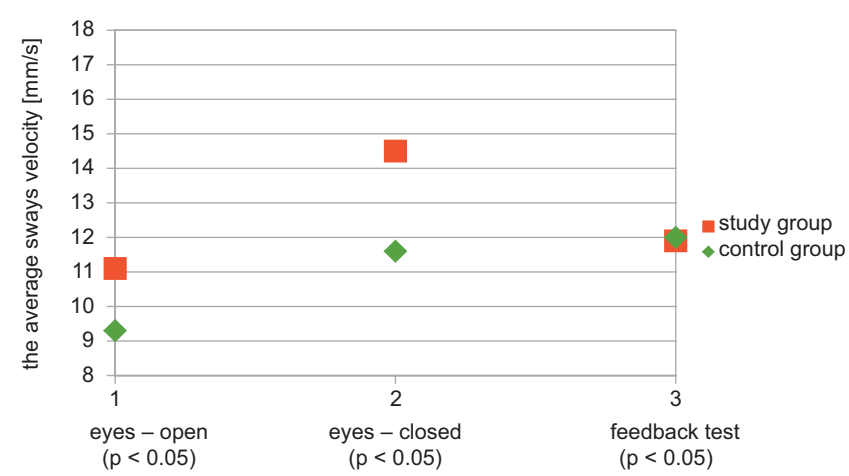

Fig. 4. The ASV in the study and control groups after ME drainage

Posturography revealed more significant vestibular system disturbances before and after ME drainage in the subgroup with mixed hearing loss compared to the subgroup with conductive hearing loss, especially before the tympanostomy procedure.

The stabilogram FDA and ASV parameters in the subgroup with conductive hearing loss after ME drainage were better in most tests in comparison to those before ME. Statistically significant values $(\mathrm{p}<0.05)$ for FDA were found with E-C and F-T (Fig. 7).

In the subgroup with mixed hearing loss, the stabilogram parameters after ME drainage were lower in all tests than those before drainage. Statistically significant values were revealed $(p<0.001)$ for FDA and ASV with E-O.

\section{Discussion}

The precise pathomechanism of balance disorders in the course of OME is not fully understood. The study conducted at the beginning of the $20^{\text {th }}$ century by Merica et al. (According to: Grace A, Pfeiderer A. Dysequilibrium and otitis media with effusion: What is the association? J Laryngol Otol. 1990;104(9):682-684) demonstrated that the functional insufficiency of the Eustachian tube causes dizziness in children. Golz et al. reported that the presence of effusion in the ME is the main cause of vertigo in children. ${ }^{12}$ One of the theories states that changes in hydrostatic pressure in the ME are transmitted through the round window and subsequently lead to secondary changes within the inner ear fluids. Due to numerous reports detecting bacteria cultures in the ME effusions, it is believed that bacterial toxins may penetrate into the labyrinth resulting in dizziness. ${ }^{15}$ The balance disorders in children with OME may also be a result of recurrent and frequent episodes of acute otitis media. Some authors report that vestibular system disturbances, but also the development of sensorineural component in OME-related hearing loss, are the result of changes within the kinocilia and stereocilia ionic channels. ${ }^{16}$

The results of our study confirm the negative effect of ME effusion on the vestibular system in children with

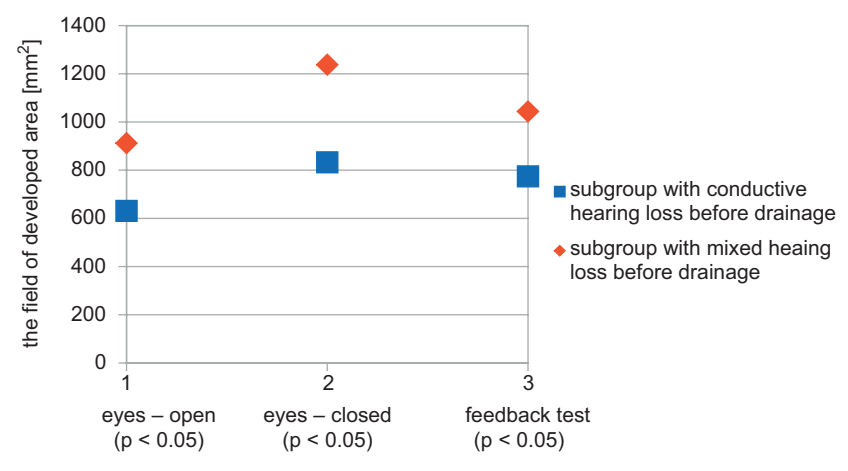

Fig. 5. The FDA in the subgroups with conductive hearing loss and with mixed hearing loss before ME drainage

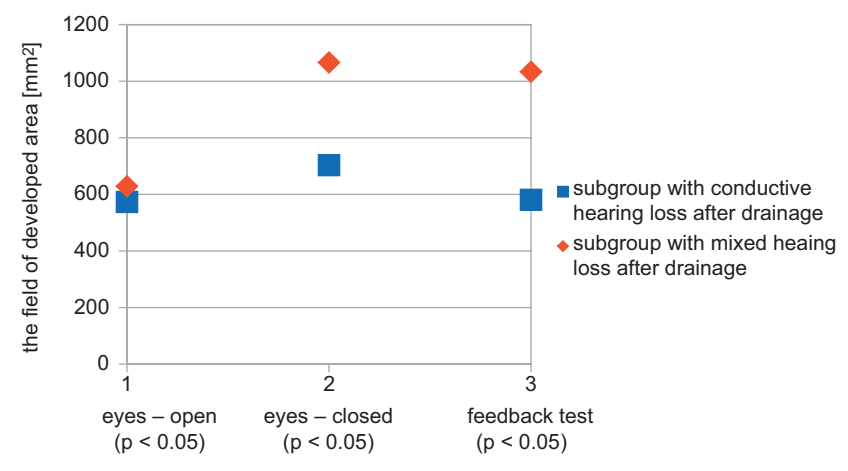

Fig. 6. The FDA in the subgroups with conductive hearing loss and with mixed hearing loss after ME drainage



Fig. 7. The FDA in the subgroup with conductive hearing loss before and after ME drainage

OME. Elevated stabilogram parameters before and after ME drainage were found in the study group in comparison with the control group. Similar results were reported by other authors studying the nature of postural stability and the effect of ME drainage on vestibulospinal reflexes in children with OME. . $^{13,14,17,18}$ After 4 weeks, there was an improvement in stabilometry parameters, though they did not completely normalize in the study period and were elevated in comparison to healthy children. Some authors emphasize the possibility of the chronic nature of vestibular system deficits, which may have a negative impact on a child's proper motor development. ${ }^{12,17}$ Casselbrant et al. evaluated the vestibular system in children with OME and studied visual dependency for balance by assessing 
the influence of optical flow on postural stability. Their study showed that maintaining postural stability in children with OME requires the excessive involvement of other, non-vestibular sensory information; this is not the case in healthy children. ${ }^{13}$

The presence of an effusion in the ME may lead to difficulties in the sensory integration, whose proper functioning is needed to develop language, and social integration. Children with vestibular dysfunction sometimes show delayed development of gross motor skill milestones compared with children without vestibular dysfunction. This emphasizes the importance of assessing balance function in children.

It was revealed that a higher number of myringotomies and ventilation tube insertions and a longer duration of OME are risk factors for developing permanent hearing loss, both conductive and sensorineural. ${ }^{19}$ Sorri et al. stated that worse thresholds, especially at high frequencies, in children with recurrent acute otitis media and OME indicate inner ear involvement. ${ }^{20}$ Mixed hearing loss associated with OME has been reported in various types of otitis media, including OME., ${ }^{9,10}$ Its precise pathogenesis is unclear. One of the theories is that inflammatory agents present in ME effusion pass through the round window membrane into the inner ear and cause temporary threshold shifts or permanent threshold shifts limited to the cochlear basal turn. ${ }^{21}$ Mutlu et al. stated that changes in the ME lead to a temporary inhibition of the vibratory movement of the oval and/or round window membrane or to an alteration of the ionic composition of the inner ear fluids. This subsequently leads to a reversible cochlear dysfunction, which usually appears as a depressed threshold region around $2 \mathrm{kHz} .{ }^{22}$ Another theory claims that mixed hearing loss is a consequence of a true disturbance of inner ear function, which does not resolve after an episode of OME. ${ }^{22,23}$ It has been revealed that elevated levels of hypoxia-inducible factor 1- $\alpha$ in ME effusion may play an important role in the pathogenesis of the bone conduction impairment associated with OME. In children with mixed hearing loss, a more significant elevation of stabilogram parameters was found compared to children with conductive hearing loss. Before ventilation tube placement, disturbances in posturographic tests were greater in children with mixed hearing than in children with conductive hearing loss. After ME drainage, the stabilogram parameters improved, though the improvement was more significant in children with conductive hearing loss than in children with mixed hearing loss, which included children with more advanced OME.

None of the parents or children had any complaints connected to vestibular organ pathology. According to the parents, their children's motor function development was normal, and no impairments in the child's psychomotor milestone achievement were reported. Interestingly, some of the parents noticed an improvement of their child's motor skills after ME drainage. The children were more willing to partake in physical activities requiring greater coordination and balance, such as climbing a ladder on the playground or riding a bicycle. This trend was also reported in the literature. ${ }^{14,15,18,24}$

\section{Conclusions}

The presence of effusion in the ME has a negative effect on the inner ear. Our findings highlight the importance of monitoring the condition of the vestibular system in all children with OME, especially in cases with mixed hearing loss and more advanced clinical stages of disease. The more pronounced improvement of vestibular function in children with conductive hearing loss after ME drainage in comparison to children with mixed hearing loss suggests that impairment of inner ear function affects its vestibular and cochlear parts.

\section{ORCID iDs}

Katarzyna Pazdro-Zastawny (D) https://orcid.org/0000-0001-8056-1198 Tomasz Zatoński (iD https://orcid.org/0000-0003-3043-4806

\section{References}

1. Rosenfeld RM, Shin JJ, Schwartz SR, et al. Clinical Practice Guideline: Otitis media with effusion executive summary (update). Otolaryngol Head Neck Surg. 2016;154(2):201-214. doi:10.1177/0194599815624407

2. Augustsson I, Engstrand I. Hearing loss as a sequel of secretory and acute otitis media as reflected by audiometric screening of Swedish conscripts. Int J Pediatr Otorhinolaryngol. 2006;70(4):703-710.

3. Kobayashi K, Kodama H, Takezawa H, Suzuki T, Kataura A. Elevation of bone conduction threshold in children with middle ear effusion. Int J Pediatr Otorhinolaryngol. 1988;16(2):95-100.

4. de Ru JA, Grote JJ. Otitis media with effusion: Disease or defense? A review of the literature. Int J Pediatr Otorhinolaryngol. 2004;68(3): 331-339.

5. Kouwen $\mathrm{H}$, van Balen FA, Dejonckere PH. Functional tubal therapy for persistent otitis media with effusion in children: Myth or evidence? Int J Pediatr Otorhinolaryngol. 2005;69(7):943-951.

6. Rinaldo A, Ferlito A. The pathology and clinical features of "glue ear": A review. Eur Arch Otorhinolaryngol. 2000;257(6):300-303.

7. Ryding $M$, White $P, K a l m ~ O$. Course and long-term outcome of "refractory" secretory otitis media. J Laryngol Otol. 2005;119(2):113-118.

8. Straetemans M, van Heerbeek N, Tonnaer E, Ingels KJ, Rijkers GT, Zielhuis GA. A comprehensive model for the aetiology of otitis media with effusion. Med Hypotheses. 2001;57(6):784-791.

9. Aviel A, Ostfeld E. Acquired irreversible sensorineural hearing loss associated with otitis media with effusion. Am J Otolaryngol. 1982; 3(3):217-222

10. Paparella MM, Goycoolea MV, Meyerhoff WL. Inner ear pathology and otitis media: A review. Ann Otol Rhinol Laryngol. 1980;89(Suppl 68):249-253.

11. Pazdro-Zastawny K, Pośpiech L, Zatoński T. Long-term evaluation of the effect of middle ear effusion on the vestibular system in children. Int J Pediatr Otorhinolaryngol. 2018;109:13-16. doi:10.1016/j. ijporl.2018.03.015

12. Golz A, Westerman ST, Gilbert LM, Joachims HZ, Netzer A. Effect of middle ear effusion on the vestibular labyrinth. $J$ Laryngol Otol. 1991;105(12):987-989.

13. Casselbrant ML, Redfern MS, Furman JM, et al. Visual induced postural sway in children with and without otitis media. Ann Otol Rhinol Laryngol. 1998;107(5 Pt 1):401-405.

14. Cohen H, Friedman EM, Lai D, Pellicer M, Duncan N, Sulek M. Balance in children with otitis media with effusion. Int J Pediatr Otorhinolaryngol. 1997;42(2):107-115. 
15. Golz A, Netzer A, Angel-Yeger B, Westerman ST, Gilbert LM, Joachims HZ Effects of middle ear effusion on the vestibular system in children. Otolaryngol Head Neck Surg. 1998;119(6):695-699.

16. Jones NS, Radomskij P, Prichard AJ, Snashall SE. Imbalance and chronic secretory otitis media in children: Effect of myringotomy and insertion of ventilation tubes on body sway. Ann Otol Rhinol Laryngol. 1990;99(6 Pt 1):477-481.

17. Gawron W, Pośpiech L, Orendorz-Fraczkowska K. An evaluation of postural stability and the effects of middle-ear drainage on vestibulo-spinal reflexes of children with chronic otitis media with effusion. Int J Pediatr Otorhinolaryngol. 2004;68(9):1175-1179.

18. Koyuncu M, Saka MM, Tanyeri Y, et al. Effects of otitis media with effusion on the vestibular system in children. Otolaryngol Head Neck Surg. 1999;120(1):117-121.

19. Ryding $M$, Konradsson $K$, White $P$, Kalm O. Hearing loss after "refractory" secretory otitis media. Acta Otolaryngol. 2005;125(3):250-255.
20. Sorri M, Maki-Torkko E, Alho OP. Otitis media and long-term followup of hearing. Acta Otolaryngol. 1995;115(2):193-195.

21. Paparella MM, Goycoolea MV, Meyerhoff WL. Inner ear pathology and otitis media: A review. Ann Otol Rhinol Laryngol Suppl. 1980;89(3 Pt 2): 249-253.

22. Mutlu C, Odabasi AO, Metin K, Basak S, Erpek G. Sensorineural hearing loss associated with otitis media with effusion. ORL J Otorhinolaryngol Relat Spec. 1992;54(2):61-65.

23. Harada T, Yamasoba T, Yagi M. Sensorineural hearing loss associated with otitis media with effusion. ORL J Otorhinolaryngol Relat Spec. 1992;54(2):61-65.

24. Golz A, Angel-Yeger B, Parush S. Evaluation of balance disturbances in children with middle ear effusion. Int J Pediatr Otorhinolaryngol. 1998;43(1):21-26. 